\title{
The Homotopy Perturbation Method for Solving the Kuramoto - Sivashinsky Equation
}

\author{
Fadhil H. Easif ${ }^{1}$, Saad A. Manaa ${ }^{2}$, Majeed A. Yousif ${ }^{3}$ \\ 1,2,3 Department of Mathematics, Faculty of Science, University of Zakho,Duhok, Kurdistan Region, Iraq
}

\begin{abstract}
The approximate solutions for the Kuramoto -Sivashinsky Equation are obtained by using the homotopy perturbation method (HPM). The numerical example show that the approximate solution comparing with the exact solution is accurate and effective and suitable for this kind of problem.
\end{abstract}

Keywords: - Homotopy Perturbation Method (HPM); Kuramoto-Sivashinsky Equation .

\section{INTRODUCTION}

The homotopy perturbation method (HPM) was first proposed by the Chinese mathematician Ji-Huan He [1-3]. Unlike classical techniques, the homotopy perturbation method leads to an analytical approximate and exact solutions of the nonlinear equations easily and elegantly without transforming the equation or linearizing the problem and with high accuracy, minimal calculation and avoidance of physically unrealistic assumptions. As a numerical tool, the method provide us with numerical solution without discretization of the given equation, and therefore, it is not effected by computation round-off errors and one is not faced with necessity of large computer memory and time. This technique has been employed to solve a large variety of linear and nonlinear problems [4- 10]. In the present study, homotopy perturbation method has been applied to solve the KuramotoSivashinsky equations. The numerical results are compared with the exact solutions. It is shown that the errors are very small.

\section{II.1 Mathematical Model}

\section{INDENTATIONS AND EQUATIONS}

The Kuramoto-Sivashinsky equation is a non-linear evolution equation and has many applications in a variety of physical phenomena such as reaction diffusion systems (Kuramoto and Tsuzuki, 1976)[11], long waves on the interface between two viscous fluids (Hooper and Grimshaw, 1985)[12], and thin hydrodynamics films (Sivashinsky, 1983)[13]. The Kuramoto-Sivashinsky equation has been studied numerically by many authors (Akrivis and Smyrlis, 2004; Manickam et al.,1998; Uddin et al., 2009)[14-16].

Consider the Kuramoto-Sivashinsky equation

Subject to the initial condition

$$
u_{t}+u u_{x}+\alpha u_{x x}+\gamma u_{x x x}+\beta u_{x x x x}=0
$$

And boundary conditions

$$
u(x, 0)=f(x) \quad a \leq x \leq b .
$$

$$
\left.\begin{array}{cc}
u(a, t)=g_{1}(t), \quad u(b, t)=g_{2}(t) & t>0 \\
\frac{\partial^{2} u}{\partial x^{2}}=h_{1}, \quad \text { at } x=a \text { and } x=b & \text { where } h_{1} \geq 0 .
\end{array}\right\}
$$

II.2 Basic idea of homotopy perturbation method

To illustrate the basic ideas of this method, we consider the following non-linear differential equation

with the following boundary conditions

$$
A(u)-f(r)=0, \quad r \in \delta
$$

$$
B\left(u, \frac{\partial u}{\partial n}\right)=0, \quad r \in \tau
$$

where $A$ is a general differential operator, $B$ a boundary operator, $f(r)$ is a known analytical function and $\tau$ is the boundary of the domain $\delta$. The operator $A$ can be decomposed into two operators, $L$ and $N$, where $L$ is a linear, and $N$ a nonlinear operator. Eq. (4) can be, therefore, written as follows:

$$
L(u)+N(u)-f(r)=0 .
$$

Using the homotopy technique, we construct a homotopy $v(r, p): \delta \times[0,1] \rightarrow R$, which satisfies:

$$
\begin{gathered}
H(v, p)=(1-p)\left[L(v)-L\left(u_{0}\right)\right]+p[A(v)-f(r)]=0, \\
p \in[0,1], r \in \delta .
\end{gathered}
$$


Or

$$
H(v, p)=L(v)-L\left(u_{0}\right)+p L\left(u_{0}\right)+p[N(v)-f(r)]=0,
$$

where $p \in[0,1]$ is an embedding parameter, $u_{0}$ is an initial approximation for the solution of Eq. (4), which satisfies the boundary conditions. Obviously, from Eqs. (7) and (8) we will have:

$$
\begin{gathered}
H(v, 0)=L(v)-L\left(u_{0}\right)=0, \\
H(v, 1)=A(v)-f(r)=0 .
\end{gathered}
$$

The changing process of $p$ form zero to unity is just that of $v(r, p)$ from $u_{0}(r)$ to $u(r)$. In topology, this is called homotopy. According to the (HPM), we can first use the embedding parameter $p$ as a small parameter, and assume that the solution of Eqs. (7) and (8) can be written as a power series in $p$ :

$$
v=v_{0}+p v_{1}+p^{2} v_{2}+\cdots
$$

Setting $p=1$, results in the approximate solution of $E q$. (1)

$$
u=\lim _{p \rightarrow 1} v=v_{0}+v_{1}+v_{2}+\cdots
$$

The combination of the perturbation method and the homotopy method is called the homotopy perturbation method (HPM), which has eliminated the limitations of the traditional perturbation methods. On the other hand, this technique can have full advantage of the traditional perturbation techniques.

The series (11) is convergent for most cases. Some criteria is suggested for convergence of the series (11), in our equation, in[1].

\section{II.3 Derivative of HPM for Kuramoto-Sivashinsky equation}

consider Kuramoto-Sivashinsky equation when

with the initial condition of

$$
u_{t}+u u_{x}+\alpha u_{x x}+\gamma u_{x x x}+\beta u_{x x x x}=0 \text {, }
$$

$$
u(x, 0)=f(x)
$$

To solve Eq. (13) by means of HPM, we construct the following homotopy for this equation:

where

$$
H(v, p)=(1-p)\left[L(v)-L\left(u_{0}\right)\right]+p[A(v)-f(r)]=0,
$$

that is

$$
H(v, p)=(1-p)\left[\frac{\partial v}{\partial t}-\frac{\partial u_{0}}{\partial t}\right]+p\left[\frac{\partial v}{\partial t}+v \frac{\partial v}{\partial x}+\alpha \frac{\partial^{2} v}{\partial x^{2}}+\gamma \frac{\partial^{3} v}{\partial x^{3}}+\beta \frac{\partial^{4} v}{\partial x^{4}}\right]=0
$$

Substituting $v$ from Eq. (8) into Eq. (13) and equating the terms with identical powers of $p$, we have

$p^{0}: \quad \frac{\partial v_{0}}{\partial t}-\frac{\partial u_{0}}{\partial t}=0, \quad v_{0}(x, 0)=f(x)$

$p^{1}: \frac{\partial v_{1}}{\partial t}+\frac{\partial v_{0}}{\partial t}+v_{0} \frac{\partial v_{0}}{\partial x}+\alpha \frac{\partial^{2} v_{0}}{\partial x^{2}}+\gamma \frac{\partial^{3} v_{0}}{\partial x^{3}}+\beta \frac{\partial^{4} v_{0}}{\partial x^{4}}=0, \quad v_{1}(x, 0)=0 ;$

$p^{2}: \frac{\partial v_{2}}{\partial t}+v_{0} \frac{\partial v_{1}}{\partial x}+v_{1} \frac{\partial v_{0}}{\partial x}+\alpha \frac{\partial^{2} v_{1}}{\partial x^{2}}+\gamma \frac{\partial^{3} v_{1}}{\partial x^{3}}+\beta \frac{\partial^{4} v_{1}}{\partial x^{4}}=0, \quad v_{2}(x, 0)=0$;

$p^{3}: \frac{\partial v_{3}}{\partial t}+v_{0} \frac{\partial v_{2}}{\partial x}+v_{2} \frac{\partial v_{0}}{\partial x}+v_{1} \frac{\partial v_{1}}{\partial x}+\alpha \frac{\partial^{2} v_{2}}{\partial x^{2}}+\gamma \frac{\partial^{3} v_{2}}{\partial x^{3}}+\beta \frac{\partial^{4} v_{2}}{\partial x^{4}}=0, \quad v_{3}(x, 0)=0 ;$

Solving these equations yields $v_{0}, v_{1}, v_{2}, v_{3}$ and so on

Thus, we can obtain

$$
u=\sum_{i=0}^{n} v_{i}=v_{0}+v_{1}+v_{2}+\cdots+v_{n}
$$

\section{III.1 Numerical Example}

\section{FIGURES AND TABLES}

In this section, we apply the technique discussed in the previous section to find numerical solution of the Kuramoto-Sivashinsky equations and compare our results with exact solutions.

Example: [17]

$$
u_{t}+u u_{x}+u_{x x}+u_{x x x x}=0, \quad x \in[0,32 \pi], \quad t \in[0,001]
$$

with the initial condition of

$$
u(x, 0)=\cos \left(\frac{x}{16}\right)\left(1+\sin \frac{x}{16}\right) ;
$$

Exact solution of problem is given by

$$
u(x, t)=\cos \left(\frac{x}{16}-t\right)\left(1+\sin \left(\frac{x}{16}-t\right)\right)
$$

$p^{0}: \quad \frac{\partial v_{0}}{\partial t}-\frac{\partial u_{0}}{\partial t}=0, \quad v_{0}(x, 0)=\cos \left(\frac{x}{16}\right)\left(1+\sin \frac{x}{16}\right) ;$ 
$p^{1}: \frac{\partial v_{1}}{\partial t}+\frac{\partial v_{0}}{\partial t}+v_{0} \frac{\partial v_{0}}{\partial x}+\frac{\partial^{2} v_{0}}{\partial x^{2}}+\frac{\partial^{4} v_{0}}{\partial x^{4}}=0, \quad v_{1}(x, 0)=0 ;$

$p^{2}: \frac{\partial v_{2}}{\partial t}+v_{0} \frac{\partial v_{1}}{\partial x}+v_{1} \frac{\partial v_{0}}{\partial x}+\frac{\partial^{2} v_{1}}{\partial x^{2}}+\frac{\partial^{4} v_{1}}{\partial x^{4}}=0, \quad v_{2}(x, 0)=0 ;$

$p^{3}: \frac{\partial v_{3}}{\partial t}+v_{0} \frac{\partial v_{2}}{\partial x}+v_{2} \frac{\partial v_{0}}{\partial x}+v_{1} \frac{\partial v_{1}}{\partial x}+\frac{\partial^{2} v_{2}}{\partial x^{2}}+\frac{\partial^{4} v_{2}}{\partial x^{4}}=0, \quad v_{3}(x, 0)=0 ;$

Then , we only find third-order term approximate solution for Eq.(22)

$$
v_{0}(x, t)=\cos \left(\frac{x}{16}\right)\left(1+\sin \left(\frac{x}{16}\right)\right)
$$

$$
\begin{aligned}
v_{1}(x, t)= & t \cos \left(\frac{x}{16}\right) \\
& \left(\frac{9200 \sin \left(\frac{x}{16}\right)-8192 \cos ^{2}\left(\frac{x}{16}\right) \sin \left(\frac{x}{16}\right)-12288 \cos ^{2}\left(\frac{x}{16}\right)+8447}{65536}\right)
\end{aligned}
$$

$$
v_{2}(x, t)=t^{2} \cos \left(\frac{x}{16}\right)
$$

$$
\begin{gathered}
\left(\frac{165437696 \sin \left(\frac{x}{16}\right)-517734400 \cos ^{2}\left(\frac{x}{16}\right) \sin \left(\frac{x}{16}\right)+201326592 \cos ^{4}\left(\frac{x}{16}\right) \sin \left(\frac{x}{16}\right)}{8589934592}\right. \\
\left.+\frac{-579706880 \cos ^{2}\left(\frac{x}{16}\right)+419430400 \cos ^{4}\left(\frac{x}{16}\right)+164855297}{8589934592}\right)
\end{gathered}
$$

$$
\begin{aligned}
& v_{3}(x, t)=t^{3} \cos \left(\frac{x}{16}\right) \\
& +\frac{4859359014912 \sin \left(\frac{x}{16}\right)-30758081134592 \cos ^{2}\left(\frac{x}{16}\right) \sin \left(\frac{x}{16}\right)}{1688849860263936} \\
& \left.+\frac{52566188621824 \cos ^{4}\left(\frac{x}{16}\right)-23570780520448 \cos ^{6}\left(\frac{x}{16}\right)+4826353967871}{1688849860263936}\right)
\end{aligned}
$$

Then approximation solution is $u(x, t)=u_{0}+u_{1}+u_{2}+u_{3}$ with third-order approximation. 
Now we compare exact solution with homotopy perturbation method (HPM) solution in Fig.1,Fig.2.
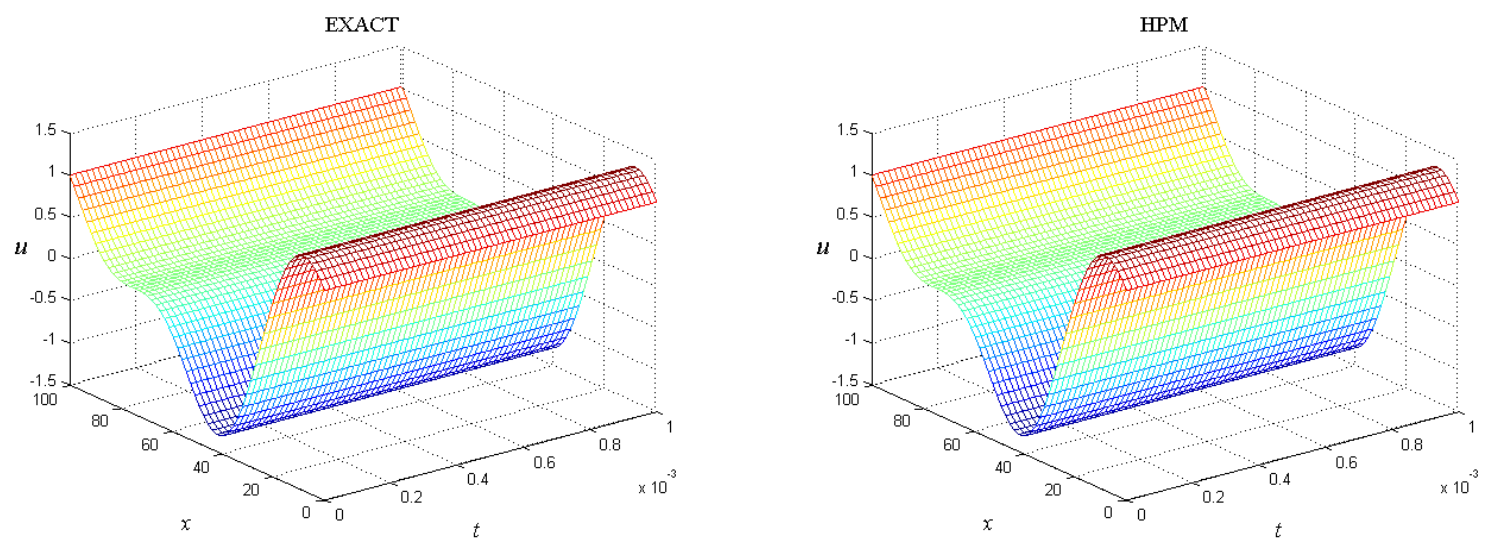

Table (1) comparison exact with homotopy perturbation method (HPM)

\begin{tabular}{|c|c|c|c|c|}
\hline$x * \pi$ & $t$ & $u_{\text {Exact }}$ & $u_{H P M}$ & $\left|u_{\text {Exact }}-u_{H P M}\right|$ \\
\hline \multirow[t]{6}{*}{0} & 0 & 1.000000000000000 & 1.000000000000000 & 0 \\
\hline & 0.0002 & 0.999799980005333 & 0.999988278219568 & $1.882982142341616 \mathrm{e}-004$ \\
\hline & 0.0004 & 0.999599920042668 & 0.999976556481800 & $3.766364391323274 \mathrm{e}-004$ \\
\hline & 0.0006 & 0.999399820144005 & 0.999964834786718 & $5.650146427130798 \mathrm{e}-004$ \\
\hline & 0.0008 & 0.999199680341350 & 0.999953113134345 & $7.534327929941131 \mathrm{e}-004$ \\
\hline & 0.001 & 0.998999500666708 & 0.999941391524700 & $9.418908579912344 \mathrm{e}-004$ \\
\hline \multirow[t]{6}{*}{6.4} & 0 & 0.602909620521184 & 0.602909620521184 & 0 \\
\hline & 0.0002 & 0.603261605525986 & 0.602924029951888 & $3.375755740976372 \mathrm{e}-004$ \\
\hline & 0.0004 & 0.603613531113795 & 0.602938440031289 & $6.750910825057410 \mathrm{e}-004$ \\
\hline & 0.0006 & 0.603965397251127 & 0.602952850759426 & $1.012546491701349 \mathrm{e}-003$ \\
\hline & 0.0008 & 0.604317203904505 & 0.602967262136334 & $1.349941768170049 \mathrm{e}-003$ \\
\hline & 0.001 & 0.604668951040459 & 0.602981674162053 & $1.687276878405974 \mathrm{e}-003$ \\
\hline \multirow[t]{6}{*}{12.8} & 0 & -1.284545252522524 & -1.284545252522524 & 0 \\
\hline & 0.0002 & -1.284489444647476 & -1.284551820710811 & $6.237606333447943 \mathrm{e}-005$ \\
\hline & 0.0004 & -1.284433528322049 & -1.284558388250076 & $1.248599280267992 \mathrm{e}-004$ \\
\hline & 0.0006 & -1.284377503541076 & -1.284564955140284 & $1.874515992073000 \mathrm{e}-004$ \\
\hline & 0.0008 & -1.284321370299405 & -1.284571521381397 & $2.501510819914454 \mathrm{e}-004$ \\
\hline & 0.001 & -1.284265128591897 & -1.284578086973377 & $3.129583814804882 \mathrm{e}-004$ \\
\hline \multirow[t]{6}{*}{19.2} & 0 & -0.333488736227371 & -0.333488736227371 & 0 \\
\hline & 0.0002 & -0.333668118536193 & -0.333484164639820 & $1.839538963727683 \mathrm{e}-004$ \\
\hline & 0.0004 & -0.333847544554259 & -0.333479593175463 & $3.679513787964717 \mathrm{e}-004$ \\
\hline & 0.0006 & -0.334027014266963 & -0.333475021834294 & $5.519924326690129 \mathrm{e}-004$ \\
\hline & 0.0008 & -0.334206527659685 & $\begin{array}{l}-0.333470450616308 \\
\end{array}$ & $7.360770433769148 \mathrm{e}-004$ \\
\hline & 0.001 & -0.334386084717798 & -0.333465879521502 & $9.202051962963198 \mathrm{e}-004$ \\
\hline \multirow[t]{6}{*}{25.6} & 0 & 0.015124368228711 & 0.015124368228711 & 0 \\
\hline & 0.0002 & 0.015095977652350 & 0.015123677789543 & $2.770013719346869 \mathrm{e}-005$ \\
\hline & 0.0004 & 0.015067621719845 & 0.015122987353990 & $5.536563414442614 \mathrm{e}-005$ \\
\hline & 0.0006 & 0.015039300412906 & 0.015122296922050 & $8.299650914345494 \mathrm{e}-005$ \\
\hline & 0.0008 & 0.015011013713235 & 0.015121606493723 & $1.105927804877852 \mathrm{e}-004$ \\
\hline & 0.001 & 0.014982761602528 & 0.015120916069009 & $1.381544664819934 \mathrm{e}-004$ \\
\hline \multirow[t]{6}{*}{32} & 0 & 1.000000000000000 & 1.000000000000000 & 0 \\
\hline & 0.0002 & 0.999799980005333 & 0.999988278219567 & $1.882982142346057 \mathrm{e}-004$ \\
\hline & 0.0004 & 0.999599920042668 & 0.999976556481800 & $3.766364391322163 \mathrm{e}-004$ \\
\hline & 0.0006 & 0.999399820144005 & 0.999964834786718 & $5.650146427135239 \mathrm{e}-004$ \\
\hline & 0.0008 & 0.999199680341350 & 0.999953113134344 & $7.534327929941131 \mathrm{e}-004$ \\
\hline & 0.001 & 0.998999500666708 & 0.999941391524699 & $9.418908579915675 \mathrm{e}-004$ \\
\hline
\end{tabular}




\section{CONCLUSION}

The (HPM) applied to Kuramoto-Sivashinsky equation and by comparing with the exact solution Fig.(1) , Fig.(2) and Table(1) shows that the absolute error is so small and the approximate solution is so closed to the exact solution.

\section{REFERENCES}

[1] J.H. He, Homotopy perturbation technique, Computer Methods in Applied Mechanics and Engineering; $178,1999,257-262$

[2] J.H. He, Homotopy perturbation method: a new nonlinear analytical technique, Applied Mathematics and Computation, 135, 2003,73-79

[3] J.H. He,Homotopy perturbation method for solving boundary value problems, Physics Letters A, 30(12), 2003,87-88.

[4] J.H. He, The homotopy perturbation method for nonlinear oscillators with discontinuities, Applied Mathematics and Computation, 151(1),2004, 287-292.

[5] J.H. He, A coupling method of a homotopy technique and a perturbation technique for non-linear problems, International Journal of Non-Linear Mechanics, 35(1), 2000, 37-43.

[6] J.H. He, Application of homotopy perturbation method to nonlinear wave equations, Chaos, Solitons \& Fractals, 26(3), 2005, 695-700.

[7] J.H. He, An approximate solution technique depending on an artificial parameter,A special example, Communications in Nonlinear Science and Numerical Simulation, 3(2), 1998, 92-97.

[8] J.H. He, 1998. A simple perturbation approach to Blasius equation, Applied Mathematics and Computation, 140(2-3), 1998, 217-222.

[9] S. Q. Wang, J.H. He, Nonlinear oscillator with discontinuity by parameter-expansion method, Chaos, Solitons \& Fractals, 35(4), 2008,688-691.

[10] D.D. Ganji, The application of He's homotopy perturbation method to nonlinear equations arising in heat transfer, Physics Letters A, 353(4-5), 2006,337-341.

[11] Kuramoto, Y., Tsuzuki, T., Persistent propagation of concentration waves in dissipative media far from thermal equilibrium, Pron. Theor. Phys. ,55, 1976, 356.

[12] Hooper, A.P., Grimshaw, R., Nonlinear instability at the interface between two viscous fluids. Phys. Fluids , 28, 1985, 37-54.

[13] Sivashinsky, G.L., Instabilities, pattern-formation, and turbulence in flames. Ann. Rev. Fluid Mech. ,15, 1983, 179-199.

[14] Akrivis, Georgios, Smyrlis, Yiorgos-sokratis, Implicit-explicit BDF methods for the KuramotoSivashinsky equation, Appl. Numer. Math. 51, 2004, 151-169.

[15] Manickam, A.V., Moudgalya, K.M., Pani, A.K., Second-order splitting combined with orthogonal cubic spline collocation method for the Kuramoto-Sivashinsky equation, Comput. Math. Appl. 35, 1998, 5-25.

[16] Uddin, M., Haq, S., Siraj-ul-Islam, A mesh-free numericalmethod for solution of the family of Kuramoto-Sivashinsky equations, Appl. Math. Comput. 212, 2009, 458-469.

[17] Ling lin, High Order Collocation Software for the Numerical Solution of Fourth Order Parabolic PDEs. , Master Thesis , Halifax, Nova.Scotia, University of Saint Mary's, 2009 